\title{
Quantitative Immunohistochemistry of Desmosomal Proteins (Plakoglo- bin, Desmoplakin and Plakophilin), Connexin-43, and N-cadherin in Ar- rhythmogenic Cardiomyopathy: An Autopsy Study
}

\author{
Fabio Tavora ${ }^{1, *}$, Mingchang Zhang ${ }^{3,4}$ Nathaniel Cresswell ${ }^{2}$, Ling $\mathrm{Li}^{1,4,5}$, David Fowler ${ }^{5}$, Marcello \\ Franco $^{1}$ and Allen Burke ${ }^{5}$ \\ ${ }^{1}$ Escola Paulista de Medicina/UNIFESP, Sao Paulo, Brazil \\ ${ }^{2}$ Georgetown University, Washington, D.C., USA \\ ${ }^{3}$ The Department of Forensic Medicine, Shanghai Medical College, Fudan University, Shanghai, China \\ ${ }^{4}$ Division of Forensic Medicine, Key Laboratory of Evidence Sciences, China University of Political Science and Law, \\ Beijing, China \\ ${ }^{5}$ University of Maryland Medical Center, Baltimore, USA
}

\begin{abstract}
Background: Arrhythmogenic right ventricular cardiomyopathy (ARVC) is a genetic disorder related to mutations in desmosomal proteins. The current study tests the hypothesis that immunohistochemical staining for desmosomal proteins is of diagnostic utility by studying autopsy-confirmed cases of ARVC.

Methods and Results: We studied 23 hearts from patients dying suddenly with ARVC. Control subject tissues were 21 hearts from people dying from non-cardiac causes $(n=15)$, dilated cardiomyopathy $(n=3)$ and coronary artery disease $(\mathrm{n}=3)$.

Areas free of fibrofatty change or scarring were assessed on 50 sections from ARVC (24 left ventricle, 26 right ventricle) and 28 sections from controls. Immunohistochemical stains against plakoglobin, plakophilin, desmoplakin, connexin-43, and $\mathrm{N}$-cadherin were applied and area expression analyzed by computerized morphometry. Desmin was stained as a control for fixation and similarly analyzed.

The mean area of desmin expression was similar in controls and ARVC (86\% vs. 85\%, p=0.6). Plakoglobin expression was $4.9 \% \pm 0.3 \%$ in controls, vs. $4.6 \% \pm 0.3 \%$ in ARVC $(\mathrm{p}=0.3)$. Plakophilin staining was $4.8 \% \pm 0.3 \%$ in controls vs. $4.4 \% \pm 03 \%$ in ARVC ( $p=0.3$ ). Desmoplakin staining was $3.4 \%$ in controls vs. $3.2 \pm 0.2 \%$ in ARVC ( $=0.6$ ). There were no significant differences when staining was compared between right and left ventricles (all $\mathrm{p}>0.1$ ).

For non-desmosomal proteins, the mean area of connexin-43 staining showed no significant difference by presence of disease.
\end{abstract}

Conclusions: The small and insignificant decrease in junction protein expression in ARVC suggests that immunohistochemistry is not a useful tool for the diagnosis.

Keywords: ARVC, arrhytmogenic cardiomyopaty, sudden death, autopsy.

\section{INTRODUCTION}

Arrhythmogenic right ventricular cardiomyopathy (ARVC), a leading cause of sudden exertional death, has been linked to a number of mutations in genes encoding cardiac desmosomal proteins [1-9]. Genes encoding desmosomal proteins that have been linked to ARVC include plakophilin2 (PKP2), followed by desmoglein-2, desmoplakin, and desmocollin-2. Decreased desmosomal protein expression has

*Address correspondence to this author at the Escola Paulista de Medicina/UNIFESP, Sao Paulo, Brazil; Tel: +558532658393;

Fax: +558532658394; E-mail: fabio.tavora@argospatologia.com.br been demonstrated in uninvolved myocardium by immunofluorescent techniques [10]. Specifically, Asimaki et al demonstrated that plakoglobin (also known as gammacatenin) was markedly decreased in uninvolved areas of myocardium in ARVC samples, with normal or variable staining for other adhesion molecules, including $\mathrm{N}$-cadherin [10]. Uninvolved areas in these studies were identified based on lack of fibro-fatty change characteristic of ARVC.

In contrast to desmosomal proteins, mutations in gap junction proteins are most frequently implicated in arrhythmias, most notably related to ion channel disorders [11,12]. A third component of the intercalated disk, the adherens 
junction, also contains proteins such as $\mathrm{N}$-cadherin, that are non-desmosomal adhesion molecules also implicated in arrhythmogenesis. Specifically, Li et al. have demonstrated a link between $\mathrm{N}$-cadherin and arrhythmias with a possible association with ARVC [13].

The purpose of the current study was to confirm the finding of abnormal desmosomal protein expression in the intercalated disc, in ARVC, by immunohistochemically staining for desmosomal proteins (two isotopes of plako-globin, plakophilin, and desmoplakin), the adherens junction protein Ncadherin and the gap junction protein connexin-43. . We used cases with available paraffin-embedded tissue from well-characterized examples of ARVC documented at autopsy published previously without immunohistochemical study [14]. We chose to use immunohistochemical techniques that are more amenable to diagnostic purposes, applying quantitative morphometric techniques to assess staining differences. The utility of immunofluorescence in diagnosing ARVC on heart biopsy has been published [10] but not yet confirmed. Immunohistochemistry has been used extensively in diagnostic pathology for quantitation of protein expression for diagnostic and prognostic purposes, and has several advantages over immunofluorescence, including ease of use, standardization, stability of the signal, and amenability to quantification of signal.

\section{MATERIALS AND METHODS}

\section{Case Selection}

Cases were chosen retrospectively, from a database of hearts seen in consultation; these have been previously published with routine histologic findings [14]. Cases diagnosed as ARVC were identified based on a diagnosis database within a 5-year period, and the blocks and slides retrieved. The diagnosis of ARVC was confirmed by the identification of fibrofatty change with degenerative myocyte changes, thinning of the right ventricle, and subepicardial fibrosis in the left ventricle, and sudden death without other cause (Fig. 1). Detailed histologic analysis with mutational analysis for PKP2 were performed and previously reported $[14,15]$. During the same time period, control hearts were chosen that approximately matched the ARVC cases by patient age, and time of consultation, with a bias to those coded as normal hearts. There were 23 cases of ARVC (aged 33 years, \pm 15 years, 16 males, 7 females), and 21 controls (mean age $34 \pm$ 12, 17 males, 4 females). Controls were 15 normal hearts (non-cardiac causes of death), 3 dilated cardiomyopathy, and 3 coronary deaths. ARVC hearts were 17 with biventricular involvement with fibrofatty change, 4 with right ventricular involvement, and 2 with left ventricular involvement only, assessed both gross and microscopically. Eight ARVC cases showed foci of inflammation, and 15 lacked inflammation. Hearts had been all examined in a similar fashion, cut unfixed, with short axis full thickness sections of left and right ventricles in each case. There were 28 sections of controls (14 right ventricle, 14 left ventricle), and 50 sections of ARVC (26 right ventricle, and 24 left ventricle). Eight of the left ventricular sections contained areas of ventricular septum. Cases were collected, fixed, and sampled all in the same manner, and fixed at least overnight before processing. Hearts were cut along short axis at $1 \mathrm{~cm}$ intervals from apex to base, and full thickness endocardial - epicardial sections of both ventricles sampled. Adequacy of fixation was tested based on light microscopy and uniform full-thickness diffuse desmin staining by immunohistochemistry.

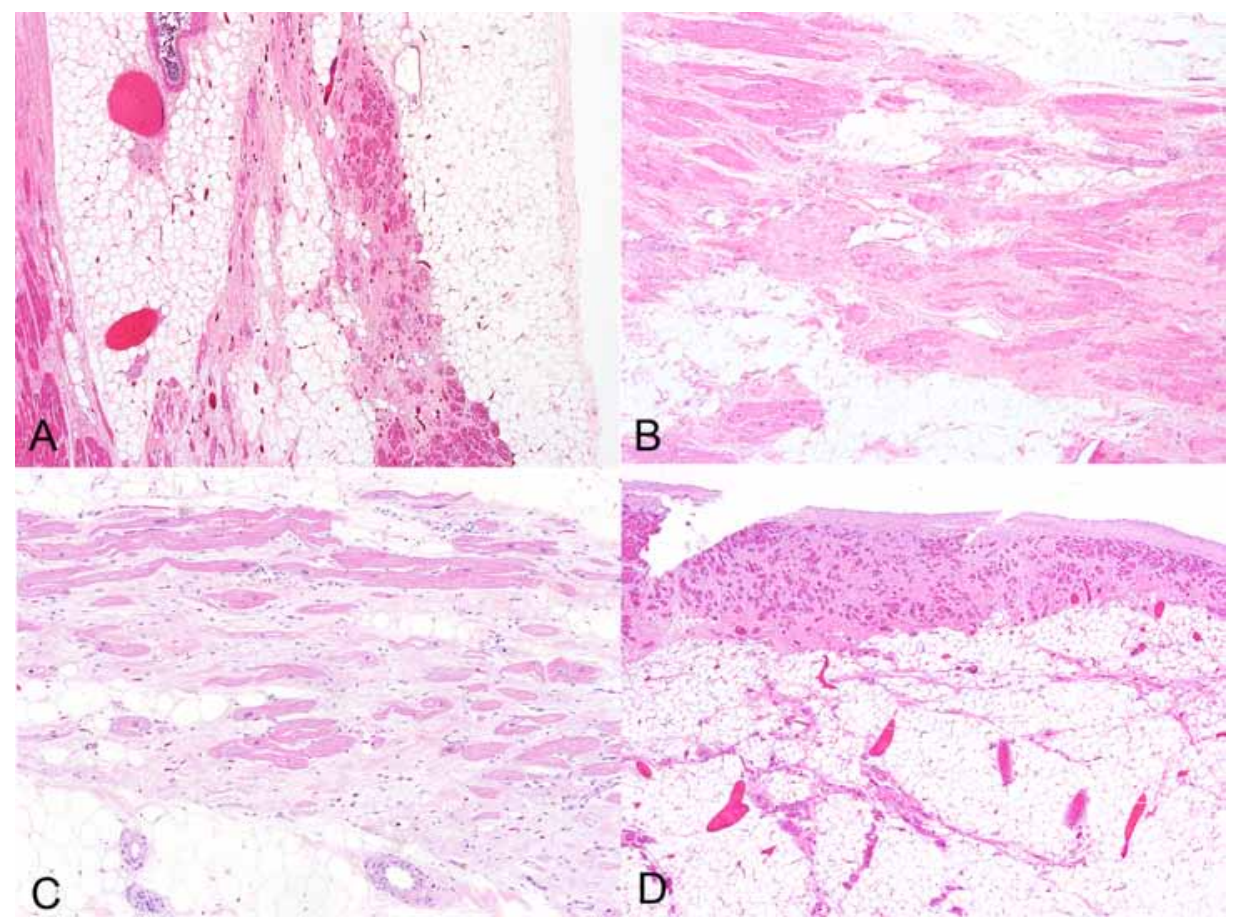

Fig. (1). ARVC, fibrofatty change. A: Section of left ventricle showing areas of fatty change with fibrosis surrounding individual myocytes. B: A section of left ventricle from a different patient, with areas of fat, randomly distribution in areas of scar and myocytes. C: A section of right ventricle single myocytes interspersed with areas of scarring and fatty change. D: Fibrofatty change in the right ventricle. Note fibrosis and fat, with collagen surrounding individual myocytes near the endocardial surface. 


\section{Immunohistochemistry}

Antibodies for desmosomal proteins were commercially obtained. These included desmoplakin 1/2 (LifeSpan Biosciences, monoclonal, 1:500, Seattle, WA), plakoglobin (gamma catenin) (Abcam, clone 15F11, 1:100, Cambridge MA), plakoglobin (JUP) (rabbit polyclonal, LifeSpan Biosciences, 1:150); and plakophilin-2 (Meridian LifeScience, Inc., monoclonal, 1:250, Saco, ME). Dilutions were determined by standard titration methods for optimal staining (strongest signal at intercalated disk with least background staining). Sources of non-desmosomal antibodies were Abcam, clone GJA1, dilution 1:2000 for connexin-43, and Abcam, clone CH19, 1:500 for n-cadherin.

Immunohistochemical studies were performed using standard techniques. Briefly, sections were deparaffinized in xylene, hydrated in graded ethanol, rinsed in distilled water, and placed in EDTA solution with steam for antigen retrieval. After serum blockade, the antibody was applied and slides were incubated in a closed water bath overnight. Slides were rinsed in PBS and secondary antibody (horse anti-mouse or goat anti-rabbit) was applied for 25 minutes. Avidin biotin complex was applied to slides for 25 minutes; the slides were rinsed and placed in hematoxylin for 2 minutes, rinsed in tap water, and then placed in ammonium hydroxide for 10 seconds and rinsed again.

\section{Morphometry}

Computed morphometry was performed using a Nuance FX ${ }^{\circledR}$ camera system. The Nuance 2.8 .0 software was used to acquire image cubes. An area of specimen at 20x magnification was chosen with longitudinally oriented myofibers based on hematoxylin eosin staining prior to immunohistochemical staining by the pathologist to ensure lack of fibrosis. The morphometric measurements were obtained in a blinded fashion. A spectral library was created from the same positive control that was used in the acquire cube protocol, as to ensure consistency. Initially, the brown chromogen was assigned a yellow spectrum (area of positivity), and the blue counterstain the region of interest (denominator, representing myocyte area, Fig. 2). The blue hematoxylin brown diaminobenzidine spectra were calculated, and the latter then assigned to red. The percentage of the region of interest was calculation with the area assigned to the red spectrum. All results were reported as percentages.

\section{Statistical Methods}

Means were compared across groups by ANOVA testing (JMP software, Cary North Carolina, USA). A p value of $<.05$ is considered significant.

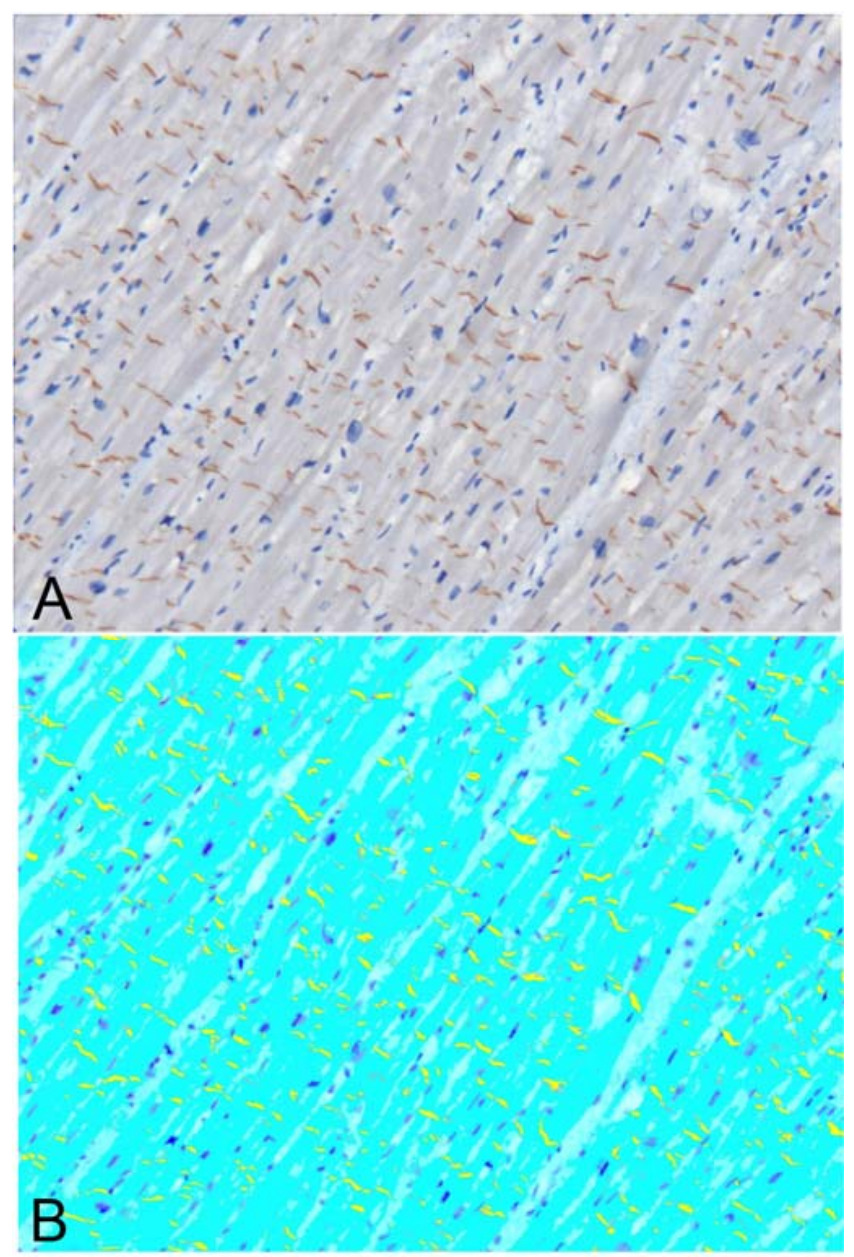

Fig. (2). A. Immunohistochemical stain for gamma-catenin in a patient with ARVC. A digitized section with intercalated discs staining with brown diaminobenzidine counterstain. B. Staining after digital subtraction colorizing brown target as yellow, for computerized assessment of percentage of staining. In this case yellow percentage of myocyte (cyan) area was rendered as 3.97\%.

\section{RESULTS}

The mean patient age was $33 \pm 15$ for the subjects and 34 \pm 13 for the controls $(p=0.4$, Table $\mathbf{1})$. None of the patients and controls had a positive toxicology or any other signs of medico-legal significance. The mean heart weight was $400 \pm$ 107 for the subjects and $419 \pm 153$ for the controls ( $\mathrm{p}=0.5$ ). Death was exertional in 15 of the 23 ARVC cases. (Table 2) Seventeen (17) ARVC cases had biventricular involvement,

Table 1. Distribution of Demographic Findings, ARVC Cases and Controls

\begin{tabular}{|c|c|c|c|}
\hline & ARVC & Controls & p \\
\hline \hline N & 23 & $17: 4$ & 0.4 \\
\hline M:F & $16: 7$ & $34 \pm 13$ & 0.8 \\
\hline Age, years mean \pm SD & $33 \pm 15$ & $419 \pm 153$ & 0.5 \\
\hline Heart weight, mean \pm SD & $400 \pm 107$ & & \\
\hline
\end{tabular}


4 were right ventricular predominant and 2 were left ventricular predominant. Details about the control cases are presented in Table 3.

Immunohistochemical studies for all the desmosomal related proteins showed localization of the stain strongly in the area of intercalated disks, with faint to absent cytoplasmic staining with desmosomal protein antibodies (Fig. 2). Desmin showed intense cytoplasmic staining in all cases. There was no difference among all the proteins between cases of ARVC (Fig. 3) and controls (Fig. 4) by percentage of positive staining analyzed in each image cube. The results

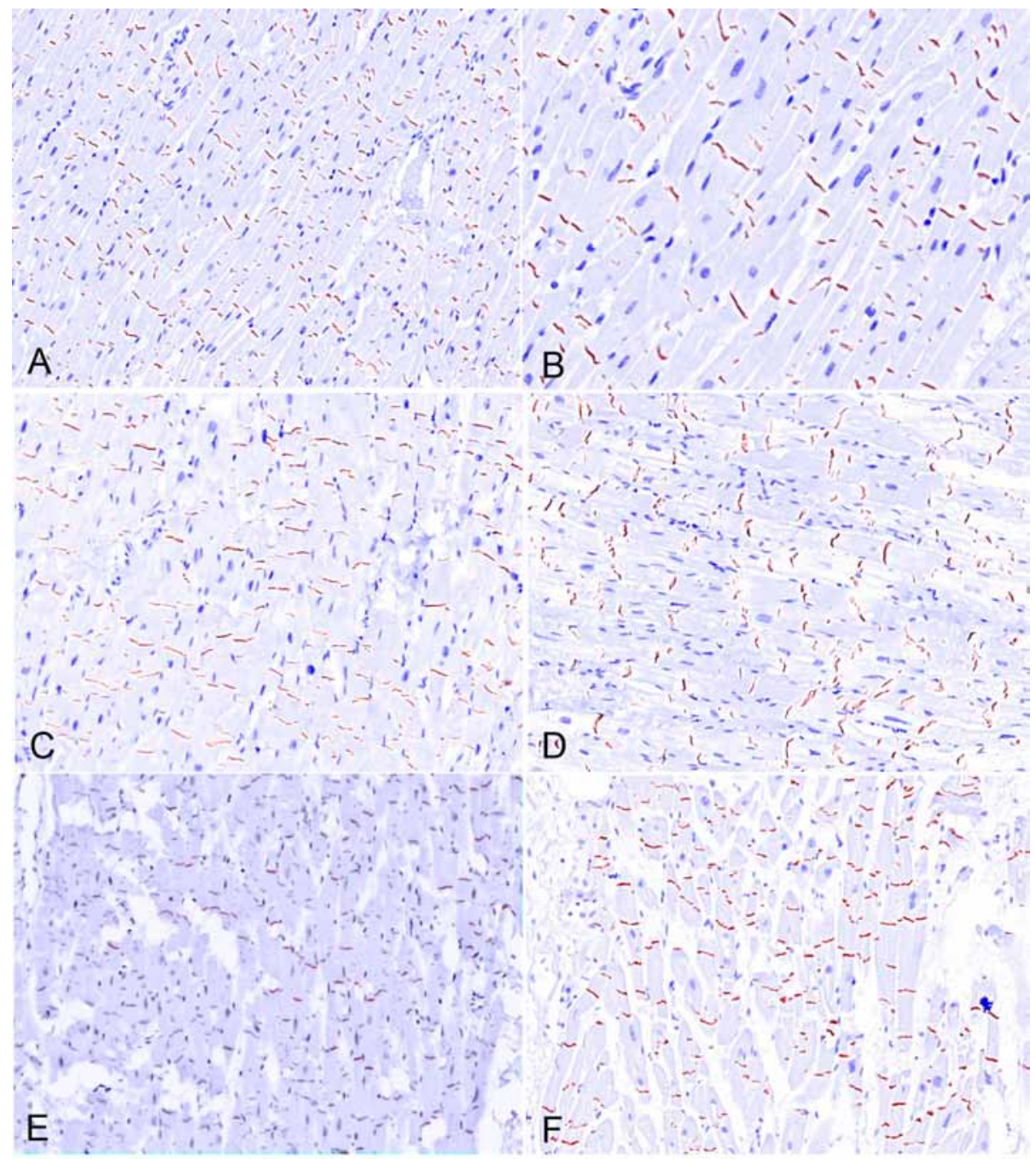

Fig. (3). Immunohistochemical staining, ARVC patients, digitized unmixed composite images, prior to color reassignment. A. Plakoglobin (antibody JUP). There is normal intensity of staining, similar to controls. B Plakoglobin (antibody JUP, higher magnification), showing normal staining intensity. B. Plakoglobin (antibody gamma catenin), showing normal intensity of staining. D. Plakophilin, showing normal intensity of staining. E. Desmoplakin, showing normal intensity of staining. F. N-cadherin, showing normal intensity of staining.

Table 2. Clinico-pathological Characteristics of ARVC Patients

\begin{tabular}{|l|c|c|c|}
\hline & N & M:F & Age \pm SD \\
\hline \hline Exertional & 15 & $12: 3$ & $33 \pm 16$ \\
Non exertional & 8 & $4: 4$ & $21 \pm 9$ \\
\hline Right ventricular* & 4 & $3: 1$ & $33+15$ \\
Biventricular & 17 & $12: 5$ & $52 \pm 1$ \\
Left ventricular* & 2 & $1: 1$ & $29 \pm 16$ \\
\hline Myocarditis present & 8 & $9: 1$ & $35 \pm 15$ \\
No myocarditis present & 15 & $9: 6$ & \\
\hline
\end{tabular}


Table 3. Clinico-pathological Characteristics of 21 Controls

\begin{tabular}{|l|c|c|c|}
\hline & N & M:F & Age \pm SD \\
\hline \hline Cardiomyopathy, dilated & 6 & $3: 3$ & $42 \pm 11$ \\
\hline $\begin{array}{l}\text { Non-cardiac deaths } \\
\text { Trauma (12) } \\
\text { Natural, non trauma (3) }\end{array}$ & 15 & $14: 1$ & $31 \pm 15$ \\
\hline
\end{tabular}

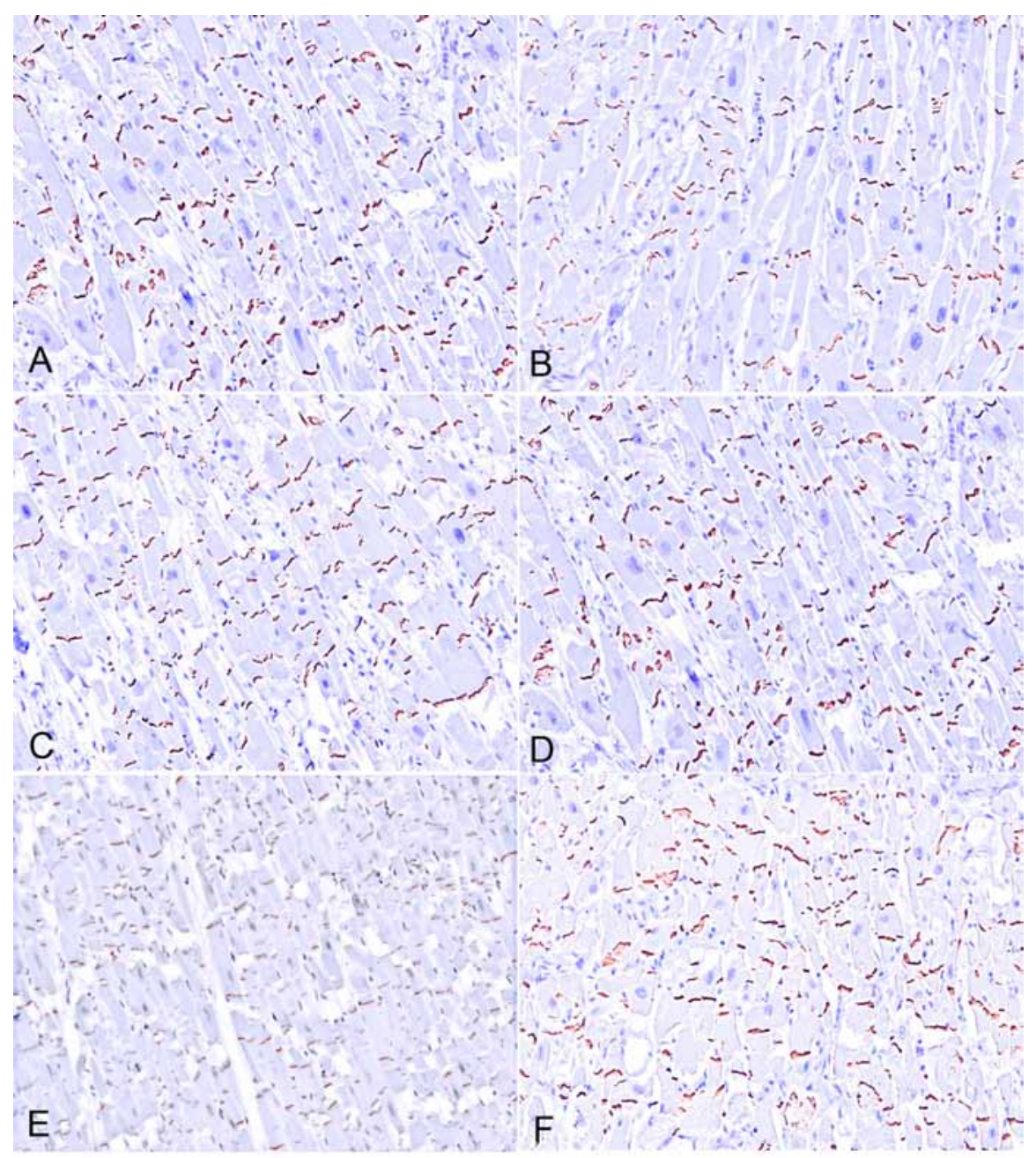

Fig. (4). Immunohistochemical staining, control patients, digitized unmixed composite images, prior to color reassignment. A. Plakoglobin (antibody JUP) showing normal intensity of staining. B Plakoglobin (antibody JUP, higher magnification) showing normal intensity of staining. B. Plakoglobin (antibody gamma catenin) showing normal intensity of staining. D. Plakophilin. showing normal intensity of staining E. Desmoplakin showing normal intensity of staining. F. N-cadherin showing normal intensity of staining.

are given in Table 4. Within each specific group, ventricular site (ARVC, controls), there was also no difference. The dilated cardiomyopathy controls showed similar staining for $\mathrm{N}$ cadherin when compared to normal controls $(\mathrm{p}=0.8)$, connexin-43 (3.8 \pm 0.6 vs. $4.0 \pm 0.5$ for controls, $\mathrm{p}=0.5$ ), desmoplakin ( $2.8 \pm 0.5$ cs. $3.5 \pm 0.3$ for controls, $\mathrm{p}=0.2$ ); gammacatenin $(\mathrm{p}=0.8)$, and JUP $(\mathrm{p}=0.8)$.

When the control groups were considered separately, as normal hearts vs. cardiac deaths, there were no significant differences in protein expression (Table 5) although there 2was a decrease in expression (not significant) in desmoplakin, connexin, and N-cadherin.

\section{DISCUSSION}

The current study assessed immunohistochemical reactivity of several proteins that reside in the intercalated disc of the human myocardium. There are 3 cell-cell connections of importance: the gap junction, or nexus; the adherens junction; and the desmosome. The gap junction is involved in ion transfer, and is composed of connexons made up of connexins, the most important in the myocardium being connexin- 
Table 4. Percentage Staining of Junctional Proteins, Controls vs. Arrhythmogenic Cardiomyopathy, Means \pm SE

\begin{tabular}{|l|c|c|c|c|c|c|c|}
\hline \multicolumn{1}{|c|}{ Antibody } & \multicolumn{3}{c|}{ Controls } & \multicolumn{3}{c|}{ Arrhythmogenic cardiomyopathy } & $\begin{array}{c}\text { P value (all, RV, } \\
\text { LV) }\end{array}$ \\
\hline & All sections & RV sections & LV sections & All sections & RV sections & LV sections & $4.8 \pm 0.3$ \\
\hline \hline Plakoglobin (JUP) & $4.8 \pm 0.3$ & $5.1 \pm 0.5$ & $4.4 \pm 0.4$ & $4.8 \pm 0.3$ & $4.6 \pm 0.3$ & $0.8,0.5,0.6$ \\
\hline Plakoglobin (gamma-catenin) & $4.9 \pm 0.2$ & $4.9 \pm 0.5$ & $4.8 \pm 0.4$ & $4.5 \pm 0.2$ & $4.6 \pm 0.3$ & $4.2 \pm 0.3$ & $0.1,0.4,0.1$ \\
\hline Plakoglobin (mean) & $4.9 \pm 0.3$ & $4.9 \pm 0.2$ & $4.6 \pm 0.3$ & $4.6 \pm 0.3$ & $4.5 \pm 0.2$ & $4.5 \pm 0.2$ & $0.6,0.3,0.8$ \\
\hline Plakophilin & $4.8 \pm 0.3$ & $4.9 \pm 0.4$ & $4.6 \pm 0.4$ & $4.4 \pm 0.3$ & $4.3 \pm 0.3$ & $4.4 \pm 0.3$ & $0.3,0.3,0.6$ \\
\hline Desmoplakin & $3.4 \pm 0.3$ & $2.4 \pm 0.4$ & $3.7 \pm 0.3$ & $3.2 \pm 0.2$ & $3.2 \pm 0.3$ & $3.2 \pm 0.3$ & $0.6,0.1,0.2$ \\
\hline N-cadherin & $4.2 \pm 0.3$ & $3.8 \pm 0.4$ & $4.2 \pm 0.3$ & $3.7 \pm 0.2$ & $3.4 \pm 0.2$ & $3.7 \pm 0.2$ & $0.2,0.4,0.2$ \\
\hline Connexin-43 & $3.7 \pm 0.3$ & $3.3 \pm 0.4$ & $4.0 \pm 0.4$ & $3.5 \pm 0.2$ & $3.3 \pm 0.3$ & $3.7 \pm 3.1$ & $0.6,0.9,0.6$ \\
\hline
\end{tabular}

Table 5. Comparison of Control Groups

\begin{tabular}{|c|c|c|c|c|}
\hline Antibody & \multicolumn{2}{|c|}{ Controls (All Sections) } & $\begin{array}{c}\text { Arrhythmogenic Car- } \\
\text { diomyopathy }\end{array}$ & $\begin{array}{l}\text { P value (Normal vs. } \\
\text { Cardiomyopathy not } \\
\text { ARVC; nl vs. ARVC) }\end{array}$ \\
\hline Plakoglobin (JUP) & $4.8 \pm 0.3$ & $4.7 \pm 0.5$ & $4.8 \pm 0.3$ & $0.9,0.7$ \\
\hline Plakoglobin (mean) & $4.8 \pm 0.3$ & $4.6 \pm 0.2$ & $4.6 \pm 0.3$ & $0.9,0.9$ \\
\hline Plakophilin & $5.0 \pm 0.4$ & $4.6 \pm 0.3$ & $4.4 \pm 0.3$ & $0.6,0.6$ \\
\hline Desmoplakin & $3.0 \pm 0.4$ & $3.8 \pm 0.4$ & $3.2 \pm 0.2$ & $0.2,0.1$ \\
\hline $\mathrm{N}$-cadherin & $3.8 \pm 0.4$ & $4.4 \pm 0.4$ & $3.7 \pm 0.2$ & $0.4,0.1$ \\
\hline
\end{tabular}

43. The adherens junction is important for structural integrity, as is the desmosome. In the myocardium, the most important transmembrane protein of the adherens junction is $\mathrm{N}$ cadherin, which is attached to beta and gamma catenin, the latter also called plakoglobin. These catenins bind the actin cytoskeleton via alpha catenin. Plakoglobin is of special interest because it is also found in the desmosome. The desmosomal armadillo proteins, plakoglobin and plakophilin, bind desmin, in contrast to those of the adherens junctions. The transmembrane proteins of the desmosome are the desmosomal cadherins desmogleins and desmocollin. They bind to each other extracellularly between adjacent cardiomyocytes, and bind intracellularly to plakoglobin and plakophilin, which, via desmoplakin, anchor desmin filaments to the cell surface.

Of the proteins in the intercalated disc, the current study assessed one gap junction protein (connexin), plakoglobin or gamma-catenin (present in the adherens junction as well as desmosome), desmoplakin (another armadillo protein of the desmosome) and plakophilin (desmosomal protein). The purpose for studying these proteins is that they have been implicated in the pathogenesis of ARVC, and have been the focus of previous immunohistochemical studies $[10,16,17]$.
The basis for linking ARVC to desmosomal proteins is partly due to the identification of mutations in desmosomal proteins in families with ARVC. As of 2007 [18], only 1 case of ARVC has been linked to mutations in plakoglobin; in this case, the underlying abnormality was actually Naxos disease, which may or may not be the same disease as ARVC. Other cases of plakoglobin mutations have been reported in cardiomyopathies with presentation similar to Naxos disease [19]. The majority of mutations in ARVC have been related to PKP2, up to $26 \%$ in some studies [20] followed by desmoplakin, desmoglein-2, and desmocollin2[21-26]. In a recent series of families with ARVC, in whom detailed imaging and clinical studies were performed, the most common mutations were present in desmoplakin, followed by plakophilin2, desmogleins and desmocollin [27].

In an immunohistochemical study of desmosomal proteins, only plakoglobin has shown to be consistently decreased in ARVC, unlike the current study [10]. Interestingly, mutations of plakoglobin have not been implicated in ARVC, other than Naxos disease. In Asimaki et al's study, [10] plakophilin-2 and desmoplakin were variably diminished in staining intensity, without clear association with mutations. N-cadherin expression was normal, with non- 
specific decreased in connexin-43 in ARVC as well as endstage heart failure. [10] Another recent study assessed the expression of plakoglobin, plakophilin, connexin-43, emerin and TMEM43 in endomyocardial biopsies of family probands [17]. The TMEM43 protein localizes to the sarcolemma and endoplasmic reticulum and mutation in its gene has been described in a small percentage of familial ARVC cases. The study showed decreased expression of plakoglobin alone without quantification, specification if the area assessed corresponded to normal or uninvolved myocardium, and the number of cases was small. The third previous study of immunolocalization of desmosomal proteins [16] showed limited specificity (57\%) for a decrease in expression of plakoglobin in diagnosing ARVC.

Unlike previous study, [10] we did not show any significant decrease in desmosomal or gap junction proteins in ARVC vs. controls. Interestingly, there was a trend suggesting decreased expression in cardiomyopathy (not ARVC) of desmoplakin, connexin, and N-cadherin, all of which were also non-significantly decreased in ARVC. These results further underscore the lack of specificity of decreased desmosome protein expression in ARVC, at least in the current study.

In our series, all cases had extensive sampling and were confirmed pathologically autopsy. Interestingly, the greatest decrease, although not statistically significant, was in the adherens junction protein $\mathrm{N}$-cadherin, which has not been shown decreased in any prior studies. Our control group did not include any end-stage heart disease patients, although 3 dilated cardiomyopathy patients had only mild decrease in desmosomal and connexin expression.

The reason for the different results may reside partly in methodology. Although the previous study [10] used primarily direct immunofluorescence, they reported a decrease in plakoglobin also with immunohistochemistry at a dilution of 1:50,000. In the study by Christensen et al. [26], the dilution used was 1:100,000 [17]. In the current study, the titration of the antibody was performed by routine clinical methods, and there was no positive staining in any case or control at a dilution of 1:50,000 using this antibody.

Our study did not show difference of protein expression with any of the antibodies between cases and controls, only a slight decrease in connexin-43 in cases of dilated cardiomyopathy. This may be partially explained by remodeling of the gap junctions following myocyte hypertrophy. The decrease, however, was not only non-significant statistically, but only seen when the image cubes were evaluated by computer morphometry. These changes were imperceptible by routine light microscopy, making it impossible as a diagnostic method. The same can be said to any decreased expression in plakoglobin, especially using non-traditional methods like hyper-diluting an antibody to thousand-fold the recommended or clinically acceptable.

As mentioned above, plakoglobin is present in the adherens junction as well as in the desmosome proper. A mutation on its gene would yield decreased protein production and/or truncated proteins with abnormal configuration. Nonetheless, the two hitherto studies that showed decreased expression of plakoglobin did not show correlation of mutation status and protein expression of plakoglobin [10,26].

Our study has the limitation of lack of mutation analyses of the studied proteins. There is an urgent need of mutation analyses in pathologically proven ARVC cases, as many studies assess families of probands that may or may not harbor the disease, or suffer from other forms of arrhythmogenic diseases.

Immunohistochemistry also has limitations: specificity of the antibodies used, fixation issues, and that it is a semiquantitative method. We addressed the observer bias and subjectivity by using a morphometric blind approach that could decrease these effects. We believe there was no significant fixation difference among cases and controls, as assessed by conventional light microscopy and the expression of desmin in all cases. Lastly, we used two different plakoglobin clones from different companies with standard immunohistochemical techniques in order to reduce the possibility of antibody specificity, arriving at similar results.

More studies are needed in order to characterize the morphologic spectrum of ARVC and the desmosomal unit may be a target for its genesis. We do not believe, however, that the mere expression of junction proteins can be diagnostic of such a complex disease, and have shown in a large series of autopsy cases that the changes in protein expression by immunohistochemistry should not be used in the clinical setting, due to lack of specificity when compared to normal controls and other cardiac diseases.

\section{CONFLICT OF INTERESTS}

Conflict of interest has not been declared by the authors.

\section{ACKNOWLEDGEMENTS}

None Declared.

\section{REFERENCES}

[1] Dalal D, Molin LH, Piccini J, et al. Clinical features of arrhythmogenic right ventricular dysplasia/cardiomyopathy associated with mutations in plakophilin-2. Circulation 2006; 113: 1641-9.

[2] Gerull B, Heuser A, Wichter T, et al. Mutations in the desmosomal protein plakophilin-2 are common in arrhythmogenic right ventricular cardiomyopathy. Nat Genet 2004; 36: 1162-4.

[3] Heuser A, Plovie ER, Ellinor PT, et al. Mutant desmocollin-2 causes arrhythmogenic right ventricular cardiomyopathy. Am J Hum Genet 2006; 79: 1081-8.

[4] Pilichou K, Nava A, Basso C, et al. Mutations in desmoglein-2 gene are associated with arrhythmogenic right ventricular cardiomyopathy. Circulation 2006; 113: 1171-9.

[5] Syrris P, Ward D, Asimaki A, et al. Desmoglein-2 mutations in arrhythmogenic right ventricular cardiomyopathy: a genotypephenotype characterization of familial disease. Eur Heart J 2007; 28: 581-8.

[6] Syrris P, Ward D, Asimaki A, et al. Clinical expression of plakophilin-2 mutations in familial arrhythmogenic right ventricular cardiomyopathy. Circulation 2006; 113: 356-64.

[7] Syrris P, Ward D, Evans A, et al. Arrhythmogenic right ventricular dysplasia/cardiomyopathy associated with mutations in the desmosomal gene desmocollin-2. Am J Hum Genet 2006; 79: 978-84.

[8] van Tintelen JP, Entius MM, Bhuiyan ZA, et al. Plakophilin-2 mutations are the major determinant of familial arrhythmogenic right ventricular dysplasia/cardiomyopathy. Circulation 2006; 113: $1650-8$. 
[9] Yang Z, Bowles NE, Scherer SE, et al. Desmosomal dysfunction due to mutations in desmoplakin causes arrhythmogenic right ventricular dysplasia/cardiomyopathy. Circ Res 2006; 99: 646-55.

[10] Asimaki A, Tandri H, Huang H, et al. A new diagnostic test for arrhythmogenic right ventricular cardiomyopathy. N Engl J Med 2009; 360: 1075-84.

[11] Ackerman MJ. State of postmortem genetic testing known as the cardiac channel molecular autopsy in the forensic evaluation of unexplained sudden cardiac death in the young. Pacing Clin Electrophysiol 2009; 32 Suppl 2: S86-9.

[12] Tester DJ, Ackerman MJ. Genetic testing for cardiac channelopathies: ten questions regarding clinical considerations for heart rhythm allied professionals. Heart Rhythm 2005; 2: 675-7.

[13] Li J, Patel VV, Radice GL. Dysregulation of cell adhesion proteins and cardiac arrhythmogenesis. Clin Med Res 2006; 4: 42-52.

[14] Tavora F, Zhang M, Franco M, et al. Distribution of biventricular disease in arrhythmogenic cardiomyopathy: an autopsy study. Hum Pathol 43(4): 592-6.

[15] Zhang M, Tavora F, Oliveira JB, et al. PKP2 mutations in sudden death from arrhythmogenic right ventricular cardiomyopathy and sudden unexpected death with negative autopsy. Circ J 2012; 76(1): 189-94

[16] Munkholm J, Christensen AH, Svendsen JH, Andersen CB. Usefulness of immunostaining for plakoglobin as a diagnostic marker of arrhythmogenic right ventricular cardiomyopathy. Am J Cardiol. 2012; 109(2): 272-5.

[17] Christensen AH, Andersen CB, Tybjaerg-Hansen A, Haunso S, Svendsen JH. Mutation analysis and evaluation of the cardiac localization of TMEM43 in arrhythmogenic right ventricular cardiomyopathy. Clin Genet 2011; 80(3): 256-64.

[18] Sen-Chowdhry S, Syrris P, McKenna WJ. Role of genetic analysis in the management of patients with arrhythmogenic right ventricular dysplasia/cardiomyopathy. J Am Coll Cardiol 2007; 50: 181321.
[19] Erken H, Yariz KO, Duman D, et al. Cardiomyopathy with Alopecia and Palmoplantar Keratoderma (CAPK) is caused by a JUP mutation. Br J Dermatol 2011; 165(4): 917-21.

[20] Awad MM, Calkins H, Judge DP. Mechanisms of disease: molecular genetics of arrhythmogenic right ventricular dysplasia/cardiomyopathy. Nat Clin Pract Cardiovasc Med 2008; 5: 258-67.

[21] Gehmlich K, Lambiase PD, Asimaki A, et al. A novel desmocollin2 mutation reveals insights into the molecular link between desmosomes and gap junctions. Heart Rhythm 2011; 8: 711-8.

[22] Cox MG, van der Zwaag PA, van der Werf C, et al. Arrhythmogenic right ventricular dysplasia/cardiomyopathy: pathogenic desmosome mutations in index-patients predict outcome of family screening: dutch arrhythmogenic right ventricular dysplasia/cardiomyopathy genotype-phenotype follow-up study. Circulation 2011; 123: 2690-700.

[23] Elliott P, O'Mahony C, Syrris P, et al. Prevalence of desmosomal protein gene mutations in patients with dilated cardiomyopathy. Circ Cardiovasc Genet 2010; 3: 314-22.

[24] Herren T, Gerber PA, Duru F. Arrhythmogenic right ventricular cardiomyopathy/dysplasia: a not so rare "disease of the desmosome" with multiple clinical presentations. Clin Res Cardiol 2009; 98: 141-58.

[25] Fidler LM, Wilson GJ, Liu F, et al. Abnormal connexin43 in arrhythmogenic right ventricular cardiomyopathy caused by plakophilin-2 mutations. J Cell Mol Med 2009; 13: 4219-28.

[26] Christensen AH, Benn M, Tybjaerg-Hansen A, Haunso S, Svendsen $\mathrm{JH}$. Missense variants in plakophilin-2 in arrhythmogenic right ventricular cardiomyopathy patients--disease-causing or innocent bystanders? Cardiology 2010; 115: 148-54.

[27] Sen-Chowdhry S, Syrris P, Ward D, Asimaki A, Sevdalis E, McKenna WJ. Clinical and genetic characterization of families with arrhythmogenic right ventricular dysplasia/cardiomyopathy provides novel insights into patterns of disease expression. Circulation 2007; 115: 1710-20.

Received: December 22, 2012

Revised: February 23, 2013

Accepted: February 24, 2013

(C) Tavora et al.; Licensee Bentham Open.

This is an open access article licensed under the terms of the Creative Commons Attribution Non-Commercial License (http://creativecommons.org/licenses/ by-nc/3.0/) which permits unrestricted, non-commercial use, distribution and reproduction in any medium, provided the work is properly cited. 